\title{
La arquitectura de Ernesto de Sola en El Salvador: del historicismo a la modernidad
}

\author{
The architecture of Ernesto de Sola in El Salvador: from \\ historicism to modernity
}

\section{Resumen}

\section{Abstract:}

Ernesto de Sola was one of the first Central American architects of the 20th century. The review of his work illustrates the consolidation of modernity in El Salvador. This paper analyzes his designs through categories as environmental adaptation and local interpretation of international languages and compares it to Latin American architecture. This research reviews multiple buildings, by means of drawings, plans, and photographs, organized in three moments: neocolonial, Art Deco, and modern, from which it is possible to extract the essential characteristics of De Sola's oeuvre: typological diversity, use of multiple architectural languages, simplification of ornamentation, structural rationalism, and adaptation to local environmental conditions. Some gaps are also evident: absence of urban planning approaches, lack of plastic integration, and technological insufficiencies restraining formal exploration. All these remain challenges for the current practice of architecture in El Salvador.

Keywords: El Salvador; Ernesto de Sola; modernity; neocolonial; Art Deco 


\section{Introducción}

Ernesto de Sola fue el primer arquitecto de El Salvador acreditado como tal (Rivas Merino, 2013; Monedero, 1970). Graduado en el Massachusetts Institute of Technology (MIT) fue uno de los representantes emblemáticos de la primera generación de profesionales de la arquitectura salvadoreña y centroamericana en el siglo $X X^{1}$. Su amplia obra, extendida a lo largo de cinco décadas, ilustra el gradual ascenso de la arquitectura moderna sobre los lenguajes historicistas tradicionales en el país. Por su volumen y visibilidad, no es exagerado decir que el trabajo del arquitecto Ernesto de Sola es parte de la memoria de El Salvador, particularmente de su ciudad capital y de algunos de sus barrios más significativos. En ese sentido, siguiendo a Aldo Rossi, estas edificaciones se han convertido en piezas de la memoria de la ciudad y se han integrado a la identidad de individuos y colectividades. Más allá de una mirada puramente descriptiva o de inventario, este artículo busca analizar el trabajo de De Sola para poner en valor su trayectoria y reconocer sus principales características y aportes a la arquitectura salvadoreña, valorando al mismo tiempo sus principales potencialidades y limitaciones. Adicionalmente, se busca trazar paralelos y continuidades con algunas de las tendencias de la arquitectura latinoamericana prevalecientes en el siglo pasado. Así se espera hacer una contribución a la historiografía de la arquitectura salvadoreña y centroamericana todavía pendientes de ser escritas.

Para ello, el artículo se organiza en cinco partes. En la primera, se explica la estrategia metodológica empleada a través de un recorrido por tres grandes conjuntos de proyectos, incluyendo análisis documental, fotográfico y dibujos de edificaciones representativas, así como una revisión de sus antecedentes personales. La segunda, tercera y cuarta parte del texto entran de lleno al análisis de los diseños agrupados en tres momentos: proyectos historicistas, edificios protomodernos y obras modernas. Finalmente, en la última parte se presentan algunas reflexiones a manera de conclusiones sobre el conjunto de la obra, sus continuidades, rupturas y lecciones aprendidas para la arquitectura salvadoreña en general.

\section{Métodos}

El estudio del trabajo de Ernesto de Sola parte de tres enfoques teóricos. Siguiendo a Arango (2012b), esta investigación se interesa por hacer una interpretación fundamentada en la alteridad y la identidad para reconocer

${ }^{1}$ Esta primera generación de arquitectos centroamericanos, formados fuera de la región, incluye personajes tales como Roberto Aycinena en Guatemala, Julio Cardenal en Nicaragua, José María Barrantes en Costa Rica o Leonardo Villanueva en Panamá. las influencias internacionales sobre el trabajo de De Sola y poner en valor el proceso de adaptación a la realidad ambiental y tecnológica local. También se recuperan los planteamientos de Segre (2003) para analizar la arquitectura del Caribe desde la categoría de "sincretismo ambiental" (p. 2). En segundo lugar, de la misma Arango (2012) se retoma el interés por entender al diseñador en un marco generacional, destacar su carácter pionero en el país y ponerlo en relación con otros creadores latinoamericanos. Ello se vincula a aproximaciones recientes como las aportadas por Esteban (2016) en su repaso de la modernidad regional con base en diseñadores, proyectos y textos. Finalmente, también se recupera de Tafuri (1997) la premisa de que el instrumento insustituible para apreciar la arquitectura es la obra en sí misma. Como dice López Pérez (2016) al retomar el método de análisis de Segre y Cárdenas, se trata de poner atención en el análisis interno de los edificios, sus códigos arquitectónicos espaciales, funcionales y técnicos. Siguiendo a Benjamin, Tafuri indicaba que dicho análisis podía hacerse de maneras táctil y óptica. La primera vinculada a la permanencia en el espacio, la segunda asociada a la contemplación de la edificación. Todo con el propósito de entender el proyecto como parte de un proceso histórico más amplio (p.161).

Algunas obras significativas de De Sola se han perdido debido a los terremotos que han afectado San Salvador por lo que su apreciación táctil es imposible, aunque perduran fotografías históricas y planos. Por otra parte, el acercamiento óptico se ha potenciado a través del ejercicio del boceto arquitectónico como herramienta analítica de aproximación e interpretación de algunos de los proyectos aquí comentados. Ello es congruente con las palabras del mismo De Sola en una carta con 25 consejos a los estudiantes de arquitectura de la Universidad de El Salvador (UES), en 1987, cuando decía "17. Dibuje sus propios planos, perspectivas, detalles, etc. usted mismo para más satisfacción, así podrá dirigir la construcción con más placer" (De Sola, 1987).

Por otra parte, estas formas de acercamiento y estudio del hecho arquitectónico se han complementado con una revisión documental del archivo de De Sola, conservado por su familia, incluyendo planos, bocetos, fotografías de época y algunos documentos escritos, lo que ha posibilitado sustentar la mirada generacional ya comentada. Además, se ha tenido acceso al material preparado para una investigación monográfica sobre la obra de De Sola en la que el autor tuvo la oportunidad de participar (Salazar et al., 2016). Todo ello como parte de una investigación académica más amplia centrada en la elaboración de una historiografía y análisis de los recursos de la producción de arquitectura en El Salvador².

Esto ha permitido desarrollar una mirada óptica, táctil y documental sobre los proyectos presentados para destacar sus cualidades centrales: organización espacial, repertorio formal, materialidad, acabados y relación con el entorno. Como lo propone Vázquez-Ramos (2017), esto se ha organizado a manera de "paseo visual", señalando las continuidades o rasgos comunes entre las obras, así como sus eventuales rupturas o novedades para inter-

${ }^{2}$ Los recursos de la producción arquitectónica en El Salvador. San Salvador: Fundación Accesarte. 
pretar las intenciones del diseñador. Dentro de cada período se destacan edificaciones específicas que condensan dichas características o que marcan singularidades en el recorrido proyectual del diseñador. Esto también permite vincular el lenguaje arquitectónico de estos edificios con el trabajo de otros diseñadores de la región en esa misma época y poner así la trayectoria de De Sola en perspectiva para, en última instancia, vincularla a la práctica actual del diseño en El Salvador. De ahí el interés en reflexionar el recorrido a partir de los temas de adaptación, interpretación e integración como lo han propuesto Segre (2003) y Arango (2012). Como diría Tafuri se trata de hacer de la historia materia proyectual en una lógica de crítica creativa (1997, p. 193).

\section{La obra arquitectónica de Ernesto de Sola}

\subsection{Algunos antecedentes}

Ernesto de Sola nació en San Salvador en 1905 y estudió en Estados Unidos entre 1928 y 1933, cuando en Centroamérica aún no existían programas universitarios de arquitectura. Obtuvo su grado académico a través de una tesis con la propuesta para un nuevo palacio del cabildo para San Salvador, el cual había sido destruido por un incendio en 1917 (Salazar et al., 2016). Trabajó en California hasta 1935, año en que regresó al país, incorporándose como arquitecto a la UES e integrándose a la empresa familiar que desde los años veinte había incursionado en el negocio inmobiliario a través del desarrollo de diversos conjuntos habitacionales en las primeras zonas de expansión de San Salvador, como el barrio Santa Anita y la colonia Flor Blanca, en las que proyectaría varias residencias privadas. De esta manera, De Sola, de forma asociada o independiente de la empresa familiar, se convertiría en un representante emblemático de la primera generación de arquitectos diseñadores y constructores del país en la que también se incluyen otras figuras nacionales, como Armando Sol, José María Durán, Salvador Choussy padre (Rivas Merino, 2013) y extranjeros, como Augusto Baratta, Brutus Targa o Bruno Capellaro (Monedero, 1970).

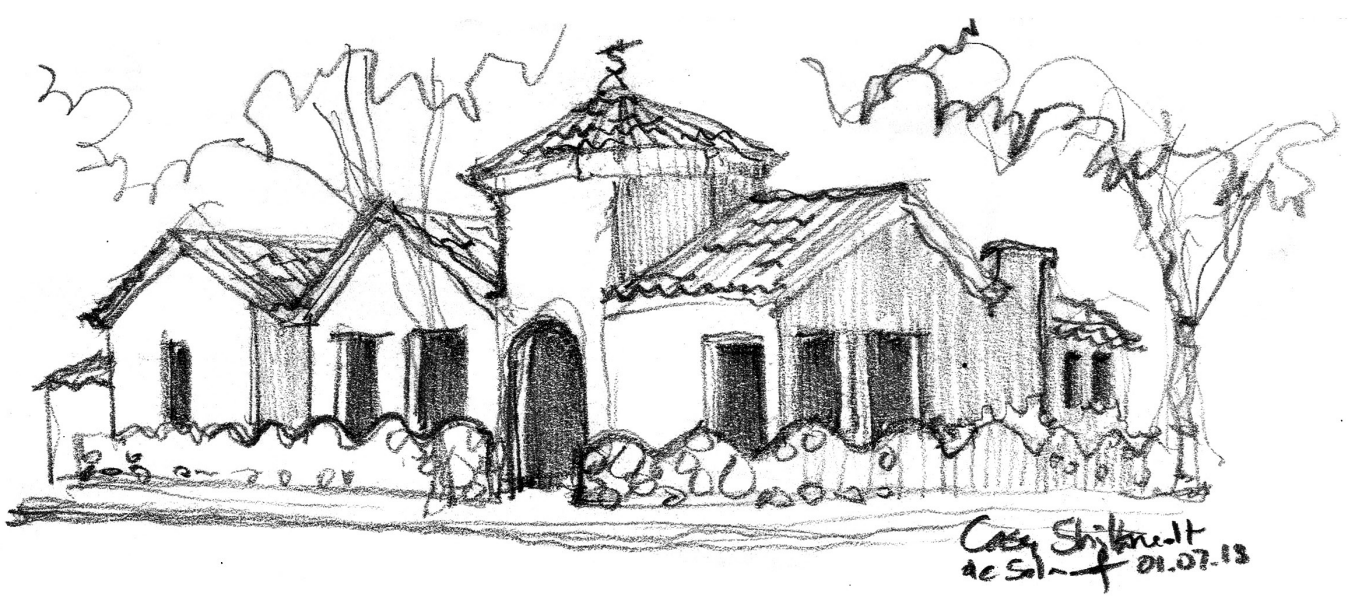

Figura 1: Boceto casa Schildknecht, Col. Flor Blanca, San Salvador

Fuente: Elaboración propia

Desde esta posición privilegiada Ernesto de Sola produjo más de 587 edificaciones en El Salvador, Guatemala y Honduras.

El recorrido por la dilatada obra de De Sola en El Salvador se organiza a partir de dos criterios complementarios: el tipológico y el lenguaje arquitectónico. En el primero, destaca una abundante producción de viviendas unifamiliares y construcciones privadas, incluyendo algunos templos religiosos y colegios. En el segundo, siguiendo la interpretación formal de Arango (2012b), sobresalen tres momentos que, en definitiva, son los que organizan el análisis: uno de corte historicista, a veces denominado neocolonial, predominante en residencias en diversos barrios y colonias de San Salvador; otro que anuncia la futura modernidad a través del art déco con numerosos proyectos comerciales, particularmente en el centro de la ciudad, y un tercero, claramente moderno, en diversos puntos de la capital, en consonancia con las tendencias internacionales. Este tipo de trayectoria diversa que abarca desde edificios historicistas hasta modernos ya ha sido señalada por autores como Segre (1999) para otros arquitectos latinoamericanos prolíficos, como el mismo Lucio Costa (p.132). De esta clasificación, que comprende momentos yuxtapuestos, resultan las categorías que se discuten a continuación.

\subsection{Proyectos historicistas}

Existen numerosas obras residenciales de Ernesto de Sola para familias acomodadas en el poniente de San Salvador. Llaman la atención por su consistencia y su localización en conjuntos homogéneos dentro de la ciudad. Hay que incluir en esta categoría casas como la Jáuregu (1942), Henríquez (1943), Argüello (1943), Menéndez (1947), Pereira (1953) y Prieto (1953), realizadas en la colonia Flor Blanca y el entorno del centro de San Salvador en la década de los cuarenta y primera mitad de los cincuenta. La casa Schildknecht (1944) (Figuras 1 y 2) pone en evidencia la organización espacial alrededor de patio interior, la importancia de los jardines exteriores y sus múltiples conexiones con los espacios interiores: salones, habitaciones y áreas de servicio, además de variaciones volumétricas en las que las cubiertas adquieren relevancia. 


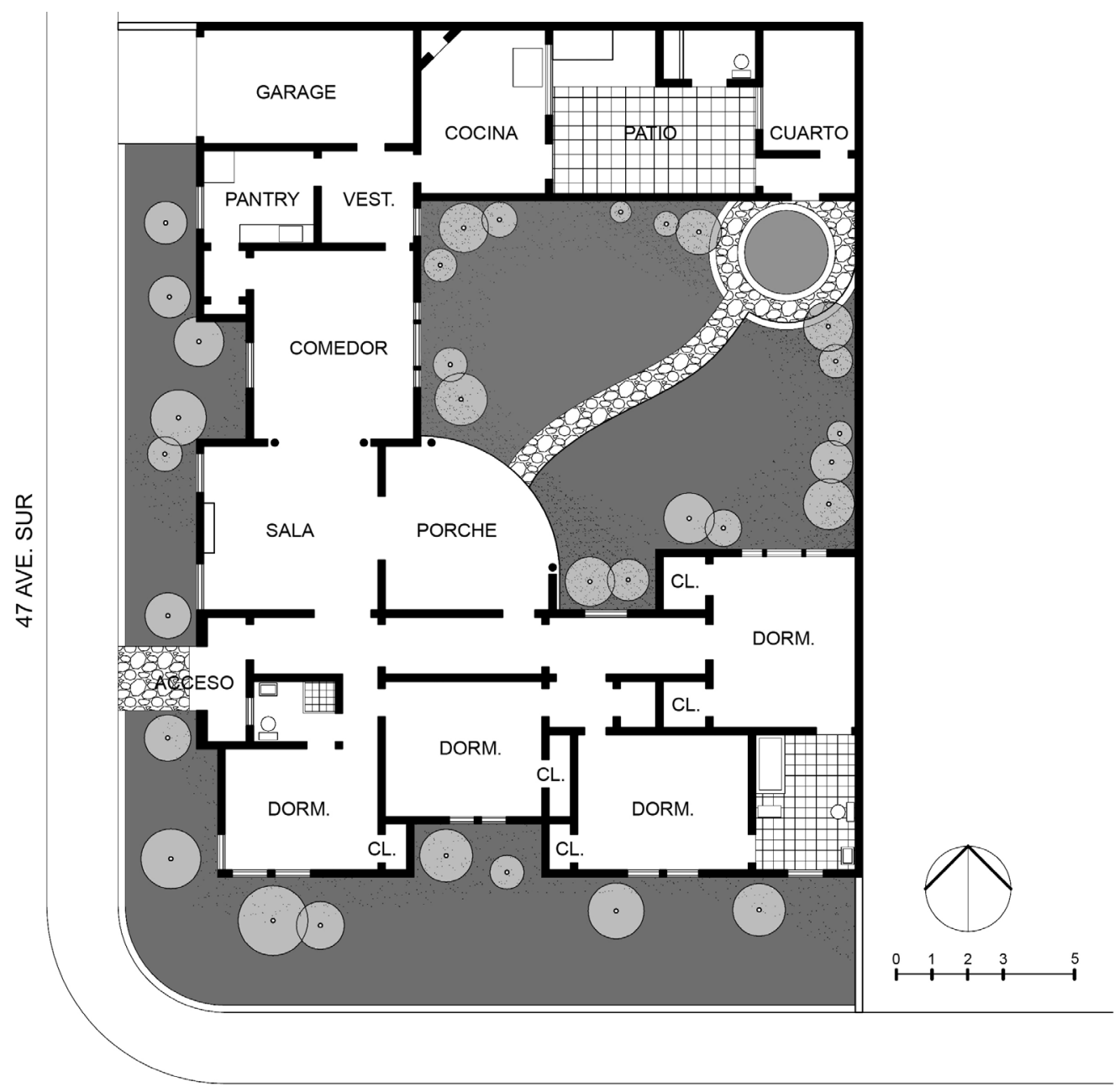

12 CALLE $P$

Figura 2: Planta arquitectónica casa Schildknecht, Col. Flor Blanca, San Salvador

Fuente: Elaboración propia en base a archivo De Sola

En la misma línea hay que resaltar las casas Kahn (1950), Carbonell (1954) en las colonias Escalón y San Benito y la casa Dueñas (1942) en el Espino. Se trata de amplias residencias con cualidades comunes, tales como: su disposición aislada en medio de terrenos amplios, jardines laterales y retiro respecto a la calle. Ello representó en su época la consolidación de la tipología alternativa de casa "tipo villa" en la ciudad, como superación de la "casa patio" heredada del modelo colonial y reiterada hasta las primeras dos décadas del siglo XX en los barrios tradicionales del centro de San Salvador (Avendaño, 2018).

A pesar de la generosidad de los espacios, las casas de esta época tienen una configuración más bien compacta, con una diversidad de espacios dispuestos en torno a la tríada de salón principal - patio interior - vestíbulo (hall) donde muchas veces destaca el protagonismo de elemento escalera y la conexión del espacio interno con el exterior a través del porche. Como lo escribió el mismo De Sola en su carta a los estudiantes de la UES: "4. Nunca olvidarse de la ventilación de la luz solar. 5. Dejar los espacios libres, como patios y jardincitos necesarios para el bienestar de los habitantes de la casa" (De Sola, 1987) Se trata de un interés por los espacios de transición: um- bral / intervalos (terraza, porche) / patio, frecuente en la arquitectura doméstica latinoamericana particularmente en el trópico húmedo (Vázquez-Ramos, 2017).

En términos volumétricos estos agrupamientos se dislocan, diferenciando los espacios por medio de sus alturas y la forma de sus cubiertas, apareciendo piezas de uno y dos niveles, cuyo contraste se acentúa por las múltiples cumbreras y las combinaciones de cubiertas a dos y cuatro aguas. Ello se complementa con alusiones coloniales como la robustez de las paredes, destacada por medio de ventanas profundas y acabados rústicos, incluyendo la piedra. También se repiten detalles como arcos de medio punto, cornisas, molduras, balaustres, aleros, azulejos y canecillos que identifican las construcciones con el lenguaje neocolonial, cuya expresión formal en Centroamérica se ha vinculado a la arquitectura tradicional de la Antigua Guatemala (Monedero, 1970).

En el fondo se trata de un código expresivo bastante practicado en América Latina en el que Segre (1999, p. 130) identifica a la vez la persistencia de un esquema compositivo académico y el inicio de una reivindicación regionalista que busca afirmarse en oposición a los len- 
guajes clásicos europeos y cuya máxima expresión se había manifestado en la Exposición Iberoamericana de Sevilla de 1929, y en obras puntuales como el Hotel Nacional de La Habana de 1930 (p.126), o el castillo Hearst en San Simeón (1919-1947). De hecho, según Segre, e neocolonial latinoamericano consiste en un repertorio formal con profundas influencias estadounidenses, particularmente de los modelos mission style y del emergente cottage suburbano, pero realizadas con las tecnologías locales, todavía artesanales, y sistemas estructurales sencillos en base a mampostería de ladrillo y cubiertas de teja. En ese sentido, se trata de una adaptación de ideas presentes en la práctica internacional, pero adaptadas a las limitaciones constructivas del país, al valor ecológico del lenguaje colonial y al gusto dominante de las élites locales. Los estudios de De Sola en la costa este de Estados Unidos y su experiencia de trabajo en la costa oeste indican un claro nexo para esta transferencia.

Dentro de este conjunto de obras, también es posible identificar algunos casos especiales en los que trasluce con mayor fuerza la matriz "historicista" de esta arquitectura habitacional, como en las residencias Sol (1949) y Nottebohm (1957). Ello se refleja en la aplicación de esquemas simétricos para organizar el espacio, el uso de cubiertas cónicas, de arcos, molduras, balaustradas, almohadillados y columnatas de manufactura neoclásica, aunque manteniendo los esquemas compactos organizados alrededor de un hall principal y amplias aperturas a los jardines por medio de porches y terrazas. Bajo los mismos parámetros destaca la casa Relyea (1949) en San Benito, la cual mantiene la amplitud de los jardines exteriores, el patio interno junto a la terraza y la simetría de cuerpo principal, aunque adoptando una referencia más palladiana con un hall circular, una escalera helicoidal y un pórtico cochera toscano en una aparente alusión a la Villa Rotonda.

En la misma lógica historicista hay que mencionar las propuestas del Colegio Sagrado Corazón (1939) y el Colegio La Asunción (1951) en el centro de San Salvador organizados alrededor de patios centrales rodeados de corredores y arcadas de medio punto en clara alusión a los claustros coloniales y plazas renacentistas. De igual forma, la Capilla de la Asunción y la iglesia del barrio Santa Anita, retoman esquemas basilicales y un repertorio formal tradicional entre románico y colonial.

A nivel de conjunto urbano merece una mención especial la casi desaparecida Colonia Bloom (1941) inmediata a centro tradicional de San Salvador y al Hospital Rosales ${ }^{3}$. Consiste en un notable conjunto de 16 casas unifamiliares entre medianeras, destinadas a alojar a los médicos del hospital, resueltas por medio de un repertorio formal similar a las residencias antes citadas, incluyendo porches, terrazas y patios, pero elaboradas en una escala más reducida y con gran consistencia urbana (Figura 3). En el fondo, se trata de un temprano proyecto de viviendas seriadas, que sin embargo logran diferenciarse $y$ adquirir individualidad a través de las diversas combinaciones de elementos formales manteniendo una relación homogénea y de proximidad a la calle

Un probable antecedente de este proyecto, por sus proporciones y acabados sencillos, se encuentra en dos viviendas gemelas en la colonia Freund (ca. 1938), al norte de la ciudad. A pesar de su tamaño reducido, en ellas se pueden identificar algunos temas espaciales compartidos con las futuras viviendas más amplias en los barrios privilegiados, tales como: la simetría, la continuidad de

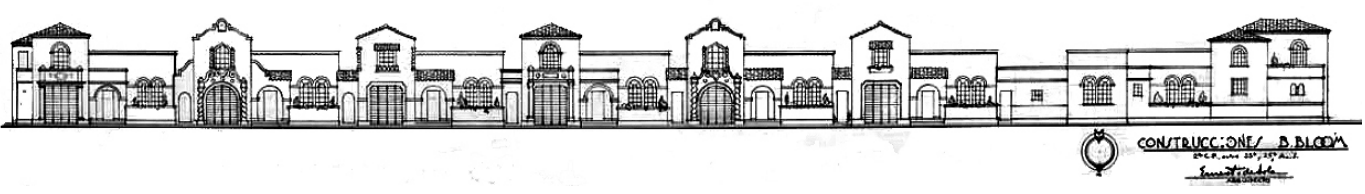

Figura 3: Fachada de conjunto de la col. Bloom

Fuente: Archivo De Sola, tratamiento digital propio

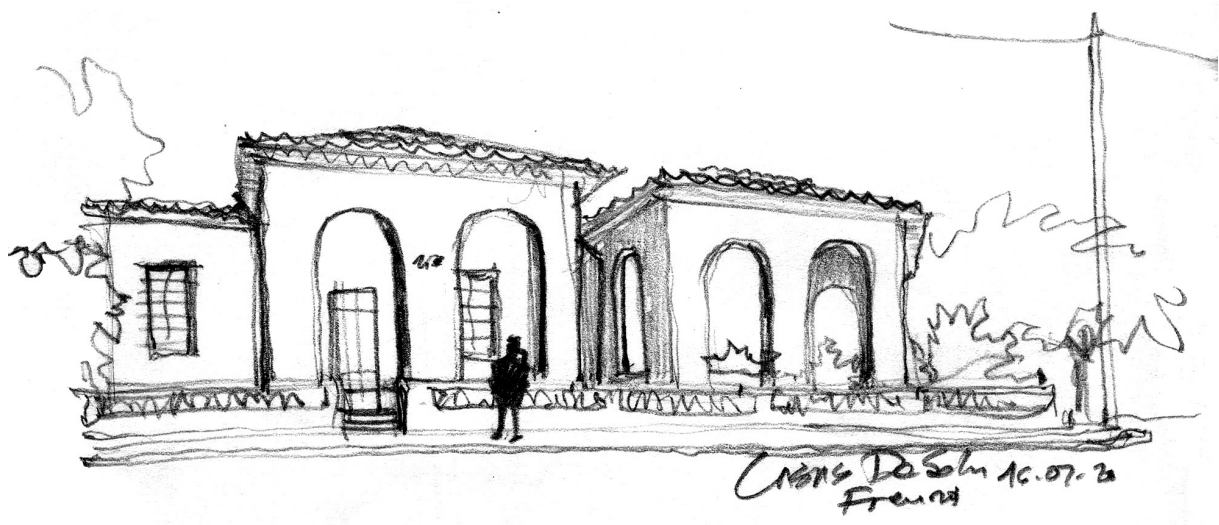

Figura 4: Boceto de casas gemelas en col. Freund, San Salvador

Fuente: Elaboración propia

${ }^{3}$ Hospital público de San Salvador 
espacio interior exterior por medio del porche, la importancia de las cubiertas y los jardines exteriores, aunque sin la aplicación de detalles historicistas y prácticamente sin elementos decorativos (Figura 4). De hecho, el primer consejo de De Sola a los futuros arquitectos era: "1. Principiar siempre por algo modesto, como por ejemplo una residencia pequeña" (De Sola, 1987).

En síntesis, este primer momento comprende un conjunto de residencias privadas y otros proyectos que superan los modelos tradicionales de la ciudad decimonónica y comparten una organización simétrica y compacta en torno a un salón principal, vestíbulo y terrazas. Son diseños que aprovechan los amplios jardines perimetrales, reivindican un acento local apropiado para las condiciones climáticas y hacen uso de lenguajes arquitectónicos del pasado, la mayoría de veces neocolonial pero también neoclásicos, siguiendo la lógica constructiva que las tecnologías locales permitían en la época.

\subsection{Edificios protomodernos y art déco}

Al mismo tiempo que producía las obras residenciales antes mencionadas, De Sola tuvo la oportunidad de diseñar diversos proyectos de "líneas más sencillas y tendencia modernizante" (Monedero, 1970 p. 213). Sobresalen cinco edificios comerciales de múltiples niveles en el centro de San Salvador: Ios ya desaparecidos Comercial (1950) y Dueñas (1947), demolidos debido a los daños causados por el terremoto de 1986 y los todavía existentes De Sola (1941), antiguo Banco Salvadoreño (1947) y Regalado (1948).

Estos edificios emblemáticos comparten varias características que relucen en los planos, dibujos y fotografías de la época. Primero, sobresale la organización del espacio a partir del manejo de un sistema de marcos estructurales regulares, que en algunos casos como en el Regalado permiten abrir espacios de ventilación e iluminación a través de patios interiores y algunas libertades formales, como la introducción de curvas. Segundo, destaca el tratamiento singular de las esquinas a través de diferentes recursos formales: desde el básico plano curvilíneo del De Sola, hasta la combinación de balcones y columnas gemelas en los edificios Dueñas y Comercial. Los numerosos bocetos para este último evidencian la preocupación del diseñador por ofrecer a la ciudad una forma singular a la esquina de la fundacional Plaza Libertad.

En términos más formales, es significativo el interés por el ritmo de llenos y vacíos de las fachadas, donde finalmente predomina el sólido en una disposición que permite la fácil lectura de los diferentes niveles y que eventualmente admite la aparición de volúmenes singulares como el núcleo de acceso - circulación vertical y puerta principal del Banco Salvadoreño (Figura 5). Como parte de esa misma lógica de composición de fachadas llama la atención el tratamiento particular del primer piso, usualmente dedicado a actividades comerciales más públicas, que suele incluir texturas y materiales diferenciados respecto del resto del edificio, y en ocasiones elementos de protección por medio de marquesinas en voladizo que cubren la acera, como en el De Sola, Dueñas y Comercial (Figura 6). Todo esto refuerza la relación edificio - calle y permite mantener la regularidad de la traza urbana, evitando los retiros y la interrupción de la histórica línea de construcción. De igual forma, en todos los casos se prevé un tratamiento especial para el coronamiento de las edificaciones, ya sea por medio de una prominente cornisa, como en el antiguo Banco Salvadoreño, o de una marquesina para la terraza de la azotea en el Comercial (Figuras 5 y 6 ).

El estudio de las fachadas permite poner en evidencia un marcado gusto por la estética lineal, acentuada por medio de elementos en relieve, incluyendo pilastras y molduras alrededor de las ventanas. Por último, se deben señalar algunos detalles de ornamentación del reperto-

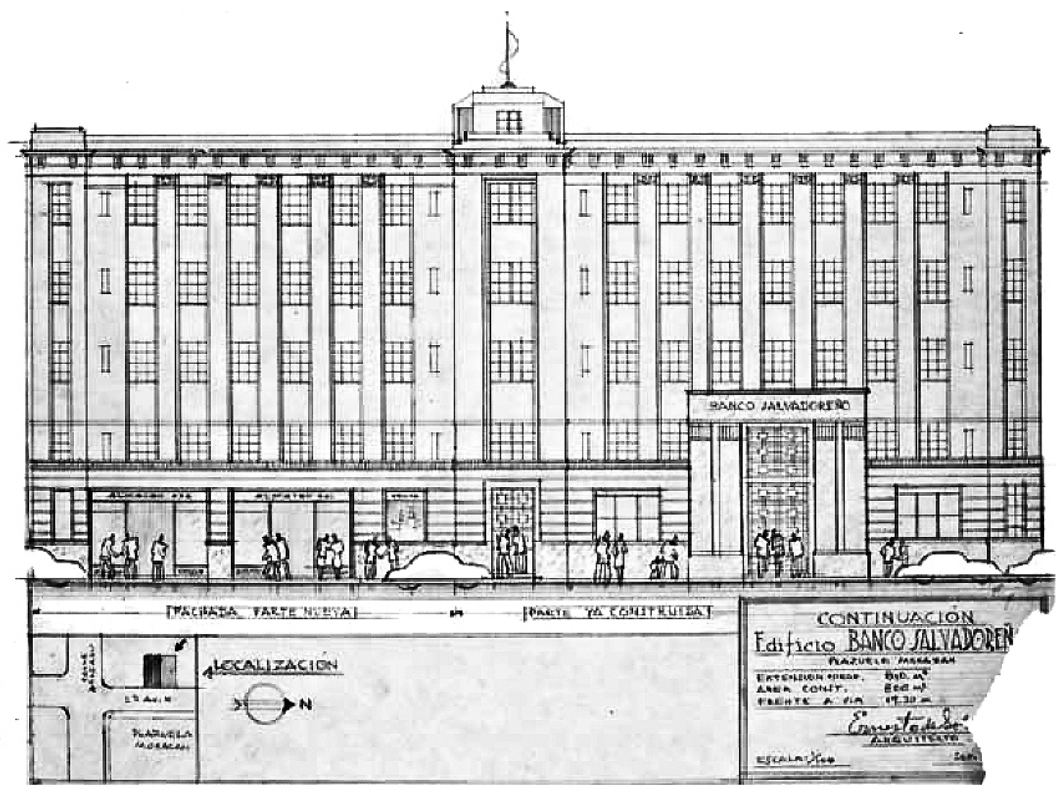

Figura 5: Fachada Banco Salvadoreño, San Salvador

Fuente: Archivo De Sola 
La arquitectura de Ernesto de Sola en El Salvador: del historicismo ala modernidad

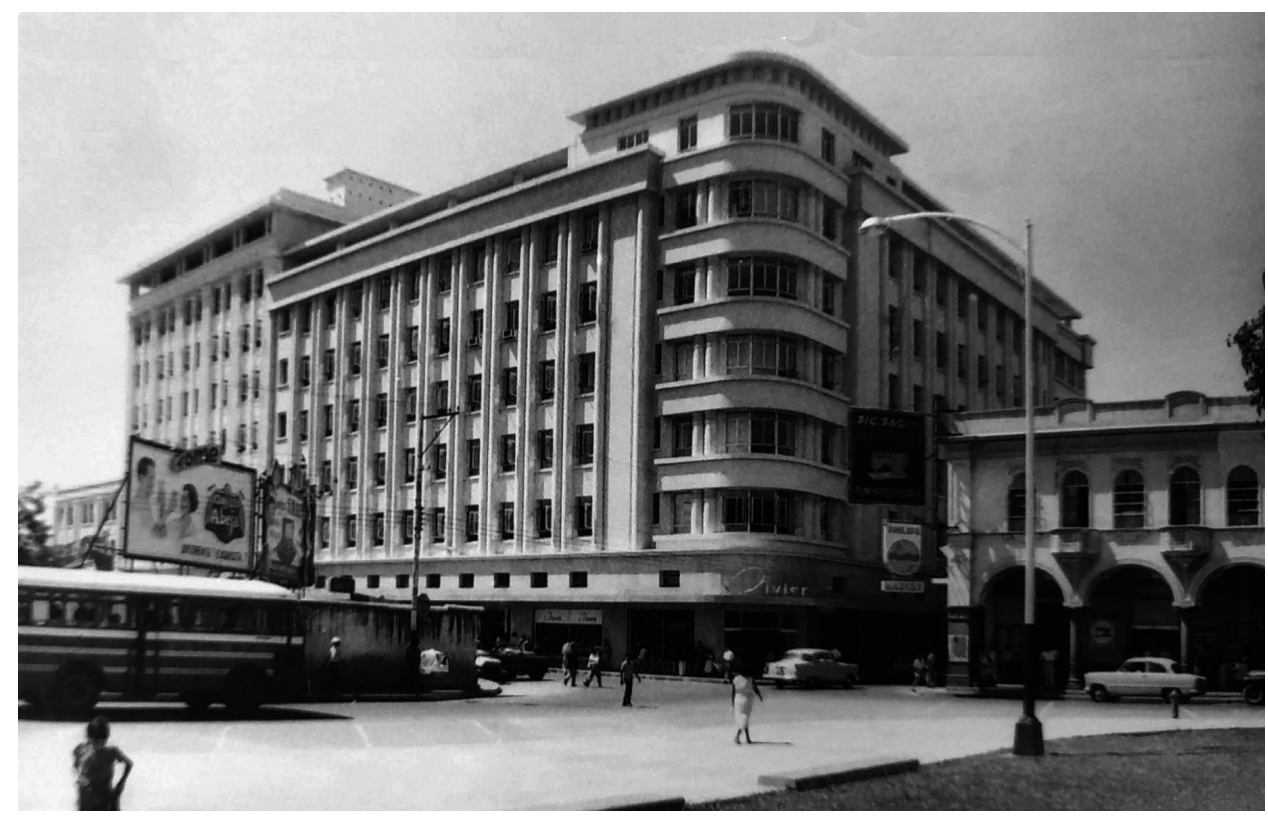

Figura 6: Esquina edificio Comercial hacia Plaza Libertad, San Salvador

Fuente: Archivo De Sola

rio art déco que exaltan la singularidad de estos edificios, tales como: el acceso principal del antiguo Banco Salvadoreño con su portal de granito verde, la puerta con elementos geométricos de bronce que abre a un vestíbulo - área de cajas a doble altura y las cornisas con grecas en alto y bajo relieve.

Al hablar de este tipo de arquitectura, que fue ocupando el Centro de San Salvador y de otras ciudades en la región entre los años cuarenta y cincuenta, Segre (2003) insiste en leer los rasgos predominantes de un art déco, heredado de Miami y difundido en todo el Caribe, que se aleja de las alusiones formales clásicas para acentuar las formas geométricas, la linealidad, los voladizos, las terrazas y el uso de formas naúticas tipo streamline que acentúan la horizontalidad de las edificaciones, pero sin entrar de lleno en el lenguaje de la modernidad vanguardista. Esta estética se refleja en el Hospital de Maternidad (1944) (Figura 7), uno de los pocos edificios públicos diseñados por De Sola y recientemente demolido, donde el volumen horizontal se remarca gracias a la profusión de franjas horizontales que funcionan como elementos de protección solar, zócalos y coronamientos, y con un contrapunto vertical en el volumen de las escaleras.

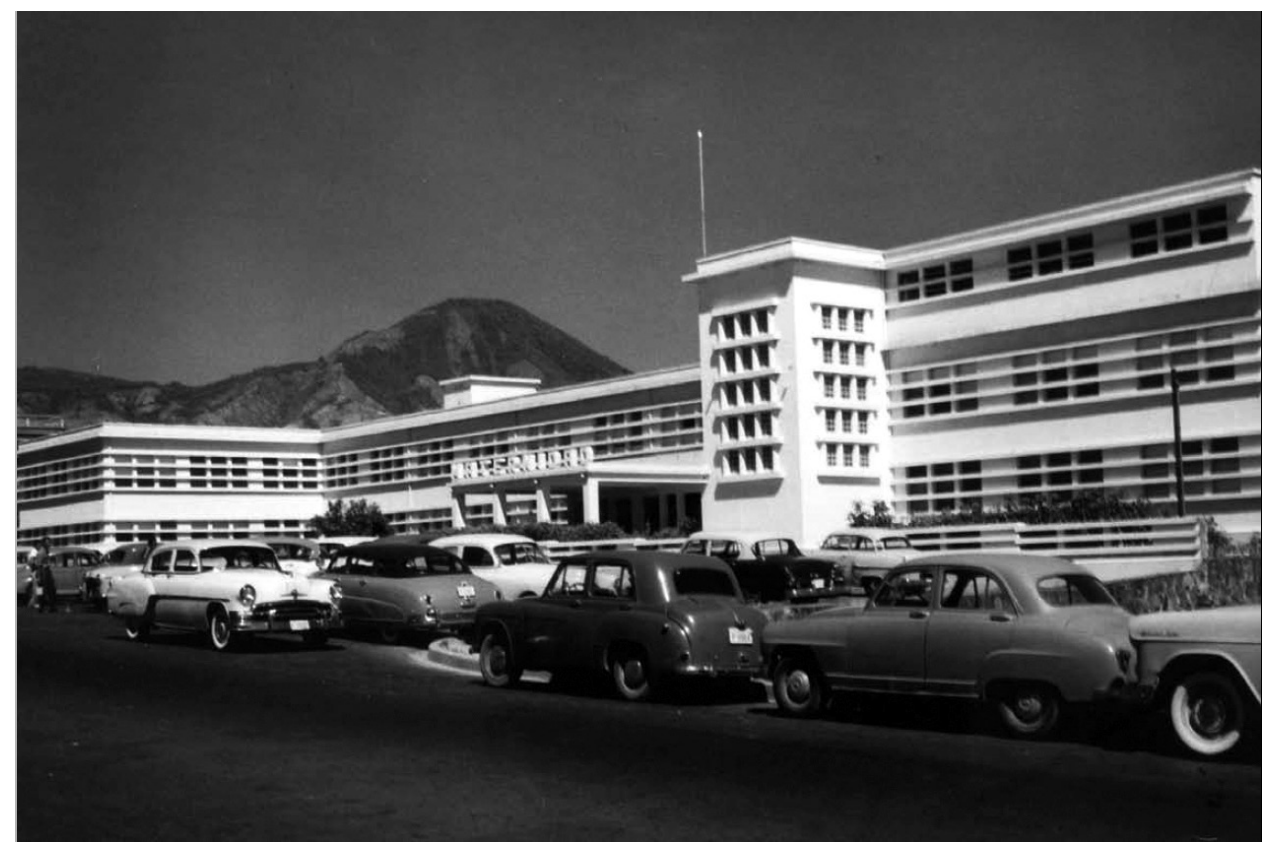

Figura 7: Hospital de Maternidad, San Salvador

Fuente: Archivo De Sola 
Utilizando la lógica de Arango (2012) y de Colquhoun (2002) se trata de una arquitectura "protomoderna": dominada por la ortogonalidad de los marcos estructurales que definen la organización espacial y formal de los proyectos, sin renunciar a los elementos ornamentales, de raíz más geométrica, y que estos autores denominan "racionalismo estructural", el cual había encontrado sus expresiones más depuradas en los rascacielos de inicios del siglo XX en Chicago y Nueva York o en la obra de diseñadores europeos como Perret. En efecto, tanto plantas como fachadas parten de la misma matriz de marcos estructurales regulares, lo que termina limitando la continuidad espacial y la planta libre más propias de la arquitectura moderna plena. Según Segre (2003) son edificios macizos que se pueden encontrar en diversas ciudades latinoamericanas, uno de cuyos exponentes centrales es el edificio Bacardí de La Habana (1929) y la obra de Federico Mariscal en México (p. 104). En resumen, se trata en San Salvador, como en otras capitales latinoamericanas, de una arquitectura de transición entre los códigos historicistas y la consolidación tardía de la visión moderna vanguardista, aunque manteniendo algunos valores urbanísticos y formales de la ciudad tradicional.

\subsection{Obras modernas}

A partir de la segunda mitad de la década del cincuenta los diseños de Ernesto de Sola adquieren una clara fisonomía moderna que evidencia significativos cambios respecto a las realizaciones previas, particularmente a través de cuatro edificios significativos: el Daglio (1956), el Magaña (1962), el IBM (1967) y La Fuente (1969).

Primero, resalta la ganancia de transparencia y liviandad por medio de la ampliación de los paños acristalados que van de piso a viga y se acercan a la lógica del muro cortina tan en boga en la arquitectura internacional de la segunda mitad del siglo XX, superando así el juego lleno- vacío del período protomoderno. Un segundo elemento que adquiere gran relevancia es el uso de cortasoles de concreto que matizan y protegen estos planos de vidrio de soleamiento, creando diversas texturas y efectos de claroscuro, en la línea que Le Corbusier, Lucio Costa y Niemeyer ya habían empleado en el Ministerio de Educación de Río de Janeiro (1945). Estos cortasoles son robustos y dominantes en la fachada poniente del Daglio (Figura 8) y mucho más livianos en el Magaña y La Fuente.

Un tercer aspecto novedoso de estas obras es la renuncia a cualquier elemento de ornamentación, incluso a los de carácter geométrico, lo que refleja un ejercicio de depuración formal del repertorio a lo largo de dos décadas. Asimismo, la paleta de materiales se reduce considerablemente a concreto, vidrio y ladrillo. En general, los acabados se limitan a superficies lisas contrastadas con planos salientes de cortasoles y estructuras para la ventanería. En cuarto lugar, los edificios modernos ponen en evidencia una nueva relación con la ciudad, probablemente vinculada a disposiciones urbanísticas. Todos, excepto el Magaña, se retranquean respecto a la línea de propiedad, dejando franjas ajardinadas que, en algunos casos, como en el Daglio, se corresponden con jardines interiores que permiten la ventilación cruzada y una mejor ambientación de los espacios de trabajo conforme a las condiciones climáticas de San Salvador. Justamente hay que destacar la calidad espacial del primer nivel del Daglio, con su doble altura y apertura hacia los dos jardines (interno y externo). Finalmente, merece especial atención la transformación de la organización estructural que se va produciendo en estos proyectos y que pasa del modelo profundamente racional del Daglio, Magaña e IBM, con marcos estructurales regulares y ortogonales, al sistema más fluido y hasta orgánico de La Fuente (Figura 9).

Vale la pena detenerse en la planta de este edificio, ajustada a la geometría curvilínea de la parcela y resuelta por medio de una serie de marcos radiales que sustentan su forma, a la vez cóncava y convexa.

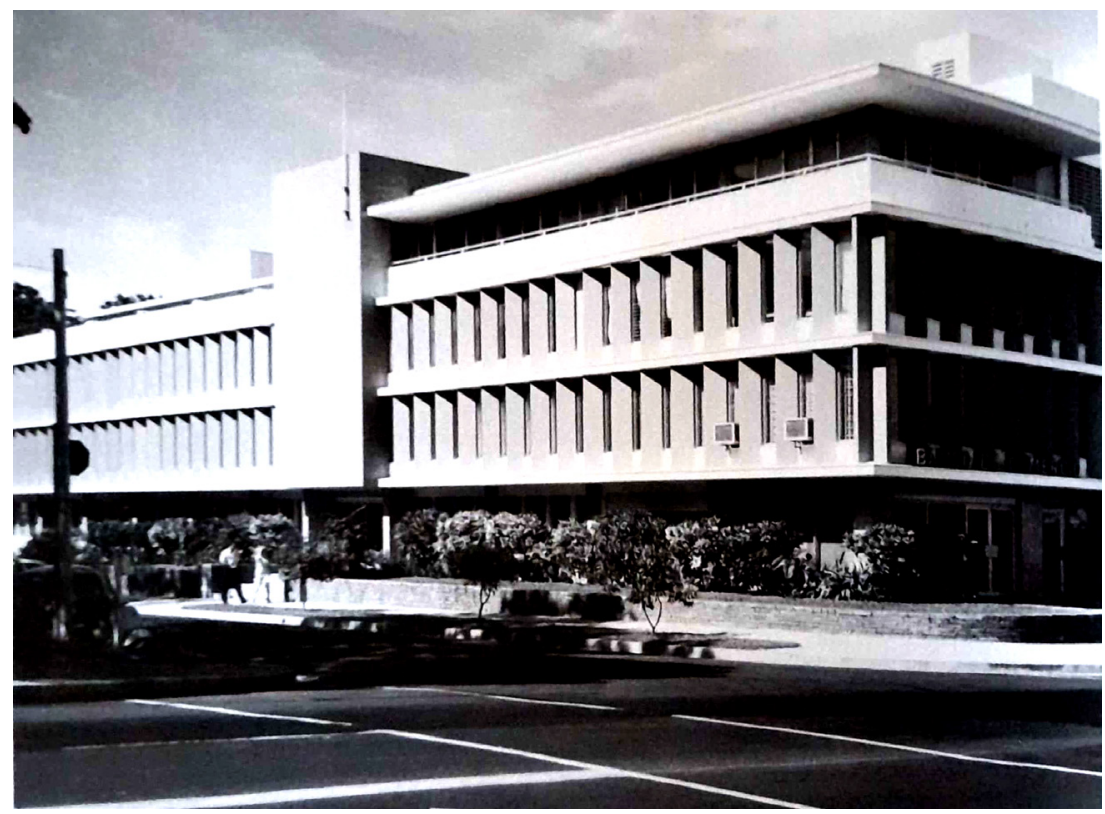

Figura 8: Edificio Daglio, San Salvador

Fuente: Archivo De Sola 


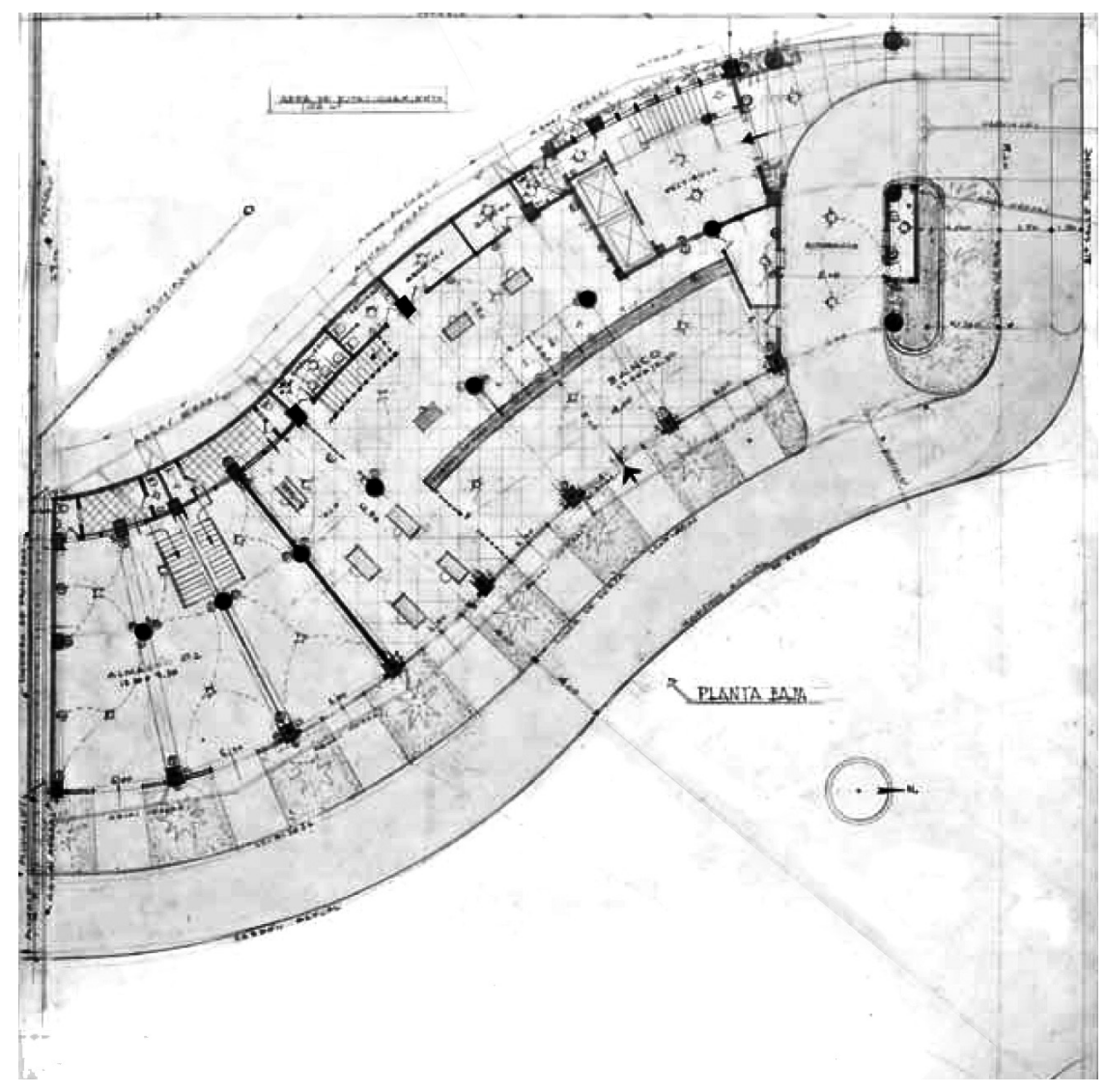

Figura 9: Planta arquitectónica edificio La Fuente, San Salvador

Fuente: Archivo De Sola y tratamiento digital propio

También destaca la resolución del autobanco y del volumen de circulaciones verticales que lo sobrevuela, en lo que parece una alusión a la plasticidad del edificio Copan de Niemeyer (Figura 10).
En síntesis, las obras postrimeras de De Sola lo ponen en la línea de la primera arquitectura moderna de El Salvador y en la lógica dominante del International Style: abandono de la ornamentación, transparencia, racionalidad

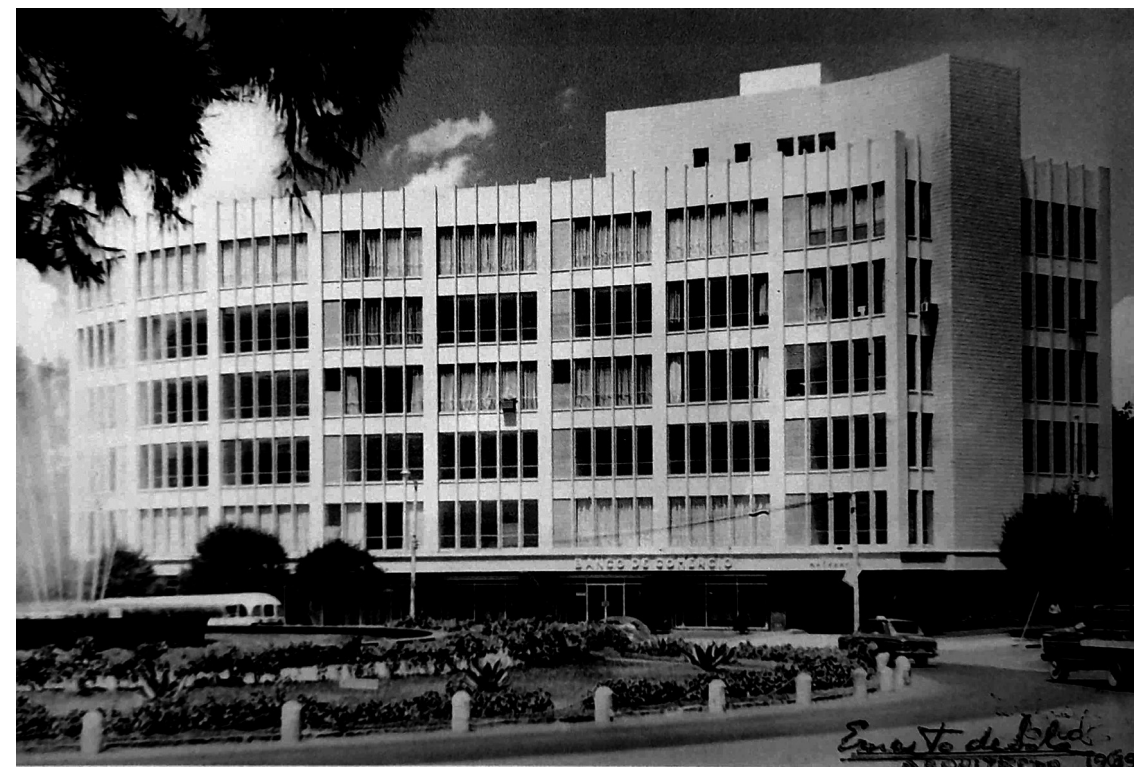

Figura 10: Edificio La Fuente, San Salvador

Fuente: Archivo De Sola y tratamiento digital propio 
estructural e incluso algunas referencias orgánicas por la incorporación de la jardinería y el control solar, lo que acentúa su relación con la realidad climática y ambiental de San Salvador, como ya se había abordado en las casas neocoloniales. No obstante, todo ello se hace sin tratar algunos temas centrales de la primera modernidad latinoamericana, como la integración plástica o la experimentación con las propiedades formales del concreto, que sí se alcanzaría en otras edificaciones modernas centroamericanas del mismo período, como el Centro Cívico de Guatemala (Fuentes, 2018), en los proyectos públicos de Terán en Managua (Martínez García, 2015), o en e anexo II de las oficinas de la Caja Costarricense del Seguro Social (CCSS) (Monge, 2016), e incluso en otras realizaciones salvadoreñas notables como las de Katstaller o Rubén Martínez (Gutiérrez, 2017). A diferencia de las residencias, los edificios modernos de De Sola no llegaron a configurar conjuntos urbanos consolidados donde los ideales del urbanismo moderno se integraran a la ciudad tradicional, como sí sucedió en México, Venezuela Brasil o Guatemala (Fuentes, 2018). Siguiendo la línea de pensamiento propuesta por Sosa y Alonso Rohner (2019) para interpretar un primer momento de la arquitectura moderna española, se trata todavía de un proceso de importación de ideas vanguardistas externas, europeas o estadounidenses, con adaptaciones propias vinculadas a las posibilidades tecnológicas existentes en el país.

\section{Conclusiones: característi- cas y limitaciones de la obra arquitectónica de Ernesto de Sola}

A través de esta revisión emergen cinco características esenciales de la obra arquitectónica de Ernesto de Sola. Primero, queda clara la diversidad de proyectos emprendidos en diferentes tipologías: residencias unifamiliares, viviendas en serie, edificios comerciales, colegios y templos religiosos. Segundo, se visualiza el uso de al menos tres lenguajes arquitectónicos diferenciados: historicis ta - neocolonial; protomoderno - art déco y moderno, con sus respectivas continuidades, rupturas y alusiones a referentes internacionales, tanto en la región latinoamericana como en Estados Unidos. Tercero, quedan en evidencia diferentes formas de tratar la ornamentación, profusa en las primeras residencias, geométrica y linea en los edificios del centro e inexistente en los edificios modernos. Cuarto, el predominio del racionalismo estructural en los edificios comerciales por medio del uso de marcos portantes ortogonales, con la notable excepción de La Fuente, de manufactura más orgánica, y quinto, el interés por algunos temas ambientales como: la apertura de las casas a los jardines por medio de porches y terrazas; el aprovechamiento de jardines exteriores e interiores; y la incorporación de elementos de protección solar como rasgos fundamentales de la forma.

En el trabajo de De Sola son notables algunas carencias todavía frecuentes en la arquitectura salvadoreña de hoy. Sobresalen: la ausencia de propuestas urbanísticas, de vivienda social y de espacios abiertos, así como el limitado desarrollo de edificios públicos, aparte del emblemático Hospital de Maternidad. En contraste, Cavalcanti (2001) al hacer su valoración de los aportes distintivos de la arquitectura moderna brasileña, señala que los primeros constituyen el deber ético del Estado, mientras que el último apunta hacia la construcción de un capital simbólico nacional (p. 14). También son llamativas la ausencia de integración con otras expresiones artísticas y las insuficiencias tecnológicas, particularmente en lo relativo al diseño estructural y construcciones de varios niveles frente al fenómeno sísmico. Estos vacíos alejan la obra de De Sola de algunos de los logros más notables de la arquitectura latinoamericana de la segunda mitad del siglo $X X$, producto de diseñadores de su misma generación. Autores como Segre (2003, 2004), Arango (2012), Rueda (2015), Esteban (2016) y Fuentes (2018), han identificado estos elementos como esenciales para caracterizar el desarrollo autónomo del diseño arquitectónico en América Latina. Ello incluye temas como: la incorporación de lo popular y vernáculo al lenguaje moderno; la adaptación del proyecto a las condiciones ambientales del lugar; la integración plástica de murales y otros elementos artísticos al edificio como parte de un discurso cultural más amplio; y una aproximación más libre a la forma a partir del dominio técnico de la tecnología del concreto y de su expresividad estructural, asociada a la visión desarrollista de la época (Browne, 2011; Martínez García, 2015).

Ello puede ser producto de una aproximación todavía muy tradicional al proceso de diseño arquitectónico que se evidencia en algunos de los consejos a los estudiantes de arquitectura de último año: "2. Dedicarse primeramente a pensar y dibujar la planta; 3 . Hacer varias calcas sobre la planta, mejorarla tomando en cuenta la distribución de los cuartos (...)" (De Sola, 1987). Esta linealidad contrasta con aproximaciones más complejas que investigadores como Hiroki y Rozestraten (2019) han documentado para figuras como Niemeyer, donde resalta la idea de proceso "abierto: sujeto a críticas, revisiones y alteraciones" (p. 16).

Lo anterior permite disponer de un marco amplio para apreciar algunos de los proyectos y períodos más significativos de la arquitectura salvadoreña del siglo $\mathrm{XX}$, justo en la transición entre los lenguajes más eclécticos e historicistas y los más racionales y claramente modernos, resaltando su valor histórico, arquitectónico y patrimonial. Queda por ver si este recorrido anuncia además algunos temas ineludibles para el futuro desarrollo de la arquitectura del país, tales como: el dominio de la escala doméstica en edificaciones de diverso tamaño; la importancia de los elementos de protección solar y climatización para establecer un vínculo con las condiciones ambientales del lugar; el interés por la relación entre interior y exterior a través de espacios de transición como terrazas, porches, patios interiores y diseños paisajistas, y finalmente el tratamiento de las referencias históricas vernáculas para acentuar el carácter local de la arquitectura sin renunciar por ello al manejo de los lenguajes internacionales. Por último, y no menos importante, habrá que saber apreciar el valor patrimonial de las ideas arquitectónicas de De Sola en la calidad de su propia producción gráfica: fotografías de la época; perspectivas a lápiz y acuarelas; planos anotados que incluyen plantas, secciones y detalles; múltiples estudios de fachada, con diversidad de opciones que denotan en su conjunto la riqueza del pro- 
ceso de diseño. Como el mismo arquitecto lo escribió en sus consejos desde una mirada casi vitruviana: "25. Por último, tenga siempre presente de que lo que usted ha diseñado y construido quedará ahí por muchos años y que nuestros hijos y nietos podrán hacer tal vez algo mejor (...) dele a su diseño nobleza, carácter y simpleza, para que su edificio hable por usted" (De Sola, 1987). El crítico estadounidense Jameson (2001, p.56) sostiene que la arquitectura es "el lenguaje estético por excelencia", y la obra arquitectónica de Ernesto de Sola pone en evidencia esta afirmación por medio de una variedad de edificios cuya presencia, memoria y análisis invita a repensar y rehacer la arquitectura salvadoreña del siglo XXI.

Cómo citar este artículo/How to cite this article: Ferrufino-Martínez, C.(2022). La arquitectura de Ernesto de Sola en El Salvador: del historicismo a la modernidad. Estoa. Revista de la Facultad de Arquitectura y Urbanismo de la Universidad de Cuenca, 11(21), 21-31. https://doi.org/10.18537/est.v011. n021.a02

\section{Referencias bibliográficas}

Arango, S. (2012). Ciudad y arquitectura. Seis generaciones que construyeron la América Latina moderna. Fondo de Cultura Económica / Consejo Nacional para la Cultura $y$ las Artes.

Arango, S. (2012b). Arquitectura Moderna Latinoamericana: el juego de las interpretaciones. Anales del Instituto de Arte Americano e Investigaciones Estéticas "Mario J. Buschiazzo" 42 (1), 39-54.

Avendaño, A. (2018). Función habitacional del patrimonio cultural en el centro histórico de San Salvador. Estoa. Revista de la Facultad de Arquitectura y Urbanismo de la Universidad de Cuenca, 7(12), 71-81.

Browne, E. (2011). Arquitectura: crítica y nueva época. STOQ.

Cavalcanti, L. (2001). Quando o Brasil era moderno: guía de arquitetura 1928-1960. Aeroplano.

Colquhoun, A. (2002). La arquitectura moderna, una historia desapasionada. Gustavo Gili.

De Sola, E. (26 de octubre de 1987). Carta a Rodrigo Alfaro. Consejos para los estudiantes de 5 año y Arquitectos. Archivo Ernesto de Sola.

Esteban Maluenda, A. (2016). La arquitectura moderna en Latinoamérica. Antología de autores, obras y textos. Editorial Reverté.

Fuentes, S. (2018). Centro Cívico de Guatemala, Patrimonio Moderno en peligro. Estoa. Revista de la Facultad de Arquitectura y Urbanismo de la Universidad de Cuenca, 7(12), 49-58.
Gutiérrez, S. (2017). Presentación del dossier: arquitectura moderna en El Salvador. Realidad: Revista de Ciencias Sociales y Humanidades, (150), 5-8.

Hiroki, J. E., y Rozestraten, A. (2019). Una revisión crítica sobre el proceso proyectual de Oscar Niemeyer. Estoa. Revista de la Facultad de Arquitectura y Urbanismo de la Universidad de Cuenca, 8(15), 9-18.

Jameson, F. (2001). Teoría de la postmodernidad. Trotta.

López Pérez, J. M. (2016). Crítica y valoración de la arquitectura moderna. El método de Roberto Segre y Eliana Cárdenas. Academia XXII, 7 (13), 95-109.

Martínez García, G. (2015). Arquitectura moderna en Nicaragua 1960-1970, una aproximación a la obra de José F. Terán Callejas. Fundación Ortiz Gurdián.

Monedero, O. (1970). Historia de la arquitectura contemporánea en El Salvador. Editorial Universitaria.

Monge, L. A. (2016). Monografía arq. Alberto Linner Díaz: monografías de arquitectos costarricenses. Colegio de Arquitectos de Costa Rica.

Rivas Merino, V. (2013). El legado gráfico arquitectónico de Armando Sol. Revista de Museología Koot\&quot, (2), 9-25.

Rueda, C. (2015). La modernidad arquitectónica tapatía: el uso de elementos y recursos de la tradición constructiva. Estoa. Revista de la Facultad de Arquitectura y Urbanismo de la Universidad de Cuenca 4(7), 29-36.

Salazar, L., Cañas, C., Ferrufino, C., y Regazzoli, J. (2016). Ernesto de Sola arquitecto. Editorial Kalina.

Segre, R. (1999). América Latina fin de milenio, raices y perspectivas de su arquitectura. Arte y Literatura.

Segre, R. (2003). Arquitectura antillana del siglo XX. Arte y Literatura.

Segre, R. (2004). Arquitectura Brasileira Contemporânea. Viana \& Mosley.

Sosa, J. A., y Alonso Rohner, E. (2019). Ida y vuelta. Influencia exterior en la arquitectura española (en la modernidad, y algunas consecuencias). Estoa. Revista de la Facultad de Arquitectura y Urbanismo de la Universidad de Cuenca, 8(16), 97-109.

Tafuri, M. (1997). Teorías e Historia de la Arquitectura. Celeste Ediciones.

Vázquez-Ramos, F. (2017). Cuadros de una exposición: promenade architecturale por la obra de Eduardo Almeida. Revista de Arquitectura, 19(2), 28-43. 